\title{
Modelo innovador para un aparador comercial usando un algoritmo competitivo imperialista
}

\author{
Yuliet Oliva-Romero ${ }^{1}$, Alberto Ochoa-Zezatti ${ }^{1}$, Ana Marcela-Herrera ${ }^{1}$, \\ Diego Alberto Oliva-Navarro ${ }^{2}$ \\ ${ }^{1}$ Universidad Autónoma de Querétaro, Campus Juriquilla, Querétaro, México \\ ${ }^{2}$ Universidad de Guadalajara, CUCEI, Departamento de Ciencias Computacionales, \\ Guadalajara, Jal., México \\ \{yulietoliva, anaherreranavarro, diegoa.olivan\}@gmail.com, alberto.ochoa@uacj.mx
}

Resumen. El artículo presenta la resolución de un problema a partir del modelado social asociado con la adecuada selección de ropa y su distribución en un aparador comercial utilizando conjunto de 87 prendas con diferentes características asociadas con una colección de moda. Se presenta un caso de estudio con respecto a la selección de 47 diferentes combinaciones posibles utilizando datos obtenidos de los patrones culturales de diversidad descritos en DuO Store y su repoblación de existencias de ropa. El objetivo de esta investigación es aplicar una solución computacional, en este caso el uso del Algoritmo Competitivo Imperialista, para resolver un problema específico. Combinado a esto, analizamos la selección de colores aplicados sobre el vestuario, el conjunto de estudios conformado por 85 prendas permitió analizar características individuales sin afectar la visualización en el aparador comercial propuesto y nos dio la oportunidad de entender la selección de colores y formas con respecto a la representación visual. Demostrando que la adecuación de características sociales y culturales especifica la correcta selección de colores. Esta investigación trata de explicar esta innovadora representación y localización de una muestra de prendas en un aparador comercial.

Palabras clave: Algoritmo competitivo imperialista, problema multiCombinatorio, reconocimiento de patrones.

\section{Innovating Model to a Dresser using an Imperialist Competitive Algorithm}

\begin{abstract}
The paper presents a problem resolved from social Modeling associated with the adequate selection of clothes and their distribution in a Dresser using a range of 87 clothes with different features associated with a fashion collection to represent the symbolic capital of it. A case of study is presented regarding to the selection of 47 different possible combinations using data obtained from the diversity cultural patterns described in DuO Store and its stock repository of clothes. The intention of this research is to apply the computational solution in this case the use of Imperialist Competitive Algorithm to resolve a specific problem, adapted from the modeled Literature about of
\end{abstract}




\begin{abstract}
Societies. Combined to this, we analyzed the selection of colors to applied on the wearing, the set of studies conformed by 85 clothes allowed to analyze individual characteristics without affecting the visualization in the proposal Dresser, and gave the opportunity us to understand the selection of colors and forms with respect the visual representation. Demonstrating that the matching of characteristic social and cultural specify the correct selection of colors; this research tries to explain this innovative representation and location of a sample of clothes on a Dresser.
\end{abstract}

Keywords: Imperialist competitive algorithm, multicombinatorial problem, pattern recognition.

\title{
1. Introducción
}

El dominio de la computación bio-inspirada está ganando gradualmente impulso en los tiempos modernos. A medida que la sociedad avanza hacia una era digital, ha habido una explosión en la cantidad de datos generados. Esta explosión de datos hace que sea cada vez más difícil extraer información, así como recopilar conocimientos mediante algoritmos estándar, debido a la creciente complejidad del análisis [1]. Encontrar la mejor solución se vuelve cada vez más difícil de identificar, no es imposible debido al gran y dinámico alcance de las soluciones y la complejidad de los cálculos. La solución óptima para un problema NP-hard es un punto en el hiperespacio n-dimensional y la identificación de la solución es computacionalmente muy cara o incluso no factible. Por lo tanto, se necesitan enfoques inteligentes para identificar soluciones de trabajo adecuadas [1].

Dentro de las metaheurísticas, los algoritmos bio-inspirados están ganando prominencia gradualmente ya que estos son inteligentes, pueden aprender y adaptarse como organismos biológicos. Estos algoritmos están llamando la atención de la comunidad científica debido a la creciente complejidad de los problemas, a un creciente rango de soluciones potenciales en hiperplanos multidimensionales, a la naturaleza dinámica de los problemas y restricciones e incompletos, probabilísticos e imperfectos cambios en las decisiones de la información. Sin embargo, el rápido desarrollos en este dominio es cada vez más difícil de rastrear debido a los diferentes algoritmos introducidos.

Los métodos de optimización evolutiva, inspirados en procesos naturales, han demostrado un buen desempeño en la solución de problemas de optimización complejos. Evidencia de esto constituyen los algoritmos genéticos (inspirados en la evolución biológica de la especie humana y otros) [2], la optimización de colonias de hormigas (basada en el esfuerzo de las hormigas para encontrar la ruta óptima en la búsqueda de la fuente de alimento) [3] y optimización de enjambre de partículas (referencia al comportamiento de las partículas en la naturaleza), son ampliamente utilizados para resolver problemas de optimización de la ingeniería [4].

El algoritmo competitivo imperialista (ICA, por sus siglas en inglés) es un nuevo método evolutivo de optimización inspirado en la competencia imperialista. Comienza con una población inicial como otros algoritmos evolutivos denominada país, que son de tipo colonizado o imperialista [5]. La competencia imperialista es la parte principal 
del algoritmo propuesto y permite que las colonias converjan al mínimo global de la función de coste. Este método es una nueva estrategia de búsqueda global, sociopolíticamente motivada, que se ha introducido recientemente para abordar diferentes tareas de optimización [6].

Algunos de los mejores países de la población son seleccionados para ser el imperialista y el resto forma las colonias de estos imperialistas. Cada país está determinado por:

$$
\text { countryi }=[\mathrm{p} 1, \mathrm{p} 1, \ldots, \mathrm{pNvar}],
$$

donde $\mathrm{N}_{\mathrm{var}}$ es la dimensión del problema a optimizar [6].

Los mejores países de la población inicial, considerando la función de costos de los mismos, son seleccionados como los imperialistas y otros países conocidos como las colonias de estos imperialistas. Los valores de variable en el país se representan como número de punto flotante. El costo de un país se encuentra evaluando la función de costo $f$ en las variables $\operatorname{var}\left[\mathrm{p}_{1}, \mathrm{p}_{2}, \ldots, \mathrm{p}_{\mathrm{N}}\right]$, por lo tanto, tenemos [5]:

$$
\cos t=f(\text { country })=\left[p_{1}, p_{1}, \ldots, p_{N v a r}\right] .
$$

Seleccionamos el $\mathrm{N}_{\mathrm{imp}}$ de los países más poderosos para formar los imperios. Las restantes Ncol de la población serán las colonias pertenecientes a los imperios. Para formar los imperios iniciales, dividimos las colonias entre los imperialistas basándonos en su poder. Para dividir las colonias entre los imperialistas proporcionalmente, se define el siguiente costo normalizado de un imperialista:

$$
\mathrm{C}_{\mathrm{n}}=\mathrm{c}_{\mathrm{n}}-\max \left(\mathrm{c}_{\mathrm{i}}\right),
$$

donde $c_{n}$ es el costo del n-ésimo imperialista y $C_{n}$ es el costo normalizado. Como resultado el poder normalizado de cada imperialista se define como:

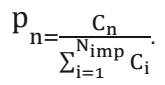

Por otra parte, el poder normalizado de un imperialista es evaluado por sus colonias. Entonces el número inicial de colonias de un imperio sería:

$$
\mathrm{NC}_{\mathrm{n}}=\operatorname{rand}\left\{\mathrm{p}_{\mathrm{n}} * \mathrm{~N}_{\mathrm{col}}\right\}
$$

La figura 1 muestra el diagrama de flujo del ICA [5].

donde, $\mathrm{NC}_{\mathrm{n}}$ es el número inicial de colonias del n-ésimo imperio y $\mathrm{N}_{\text {col }}$ es el número de total de colonias. Después de dividir las colonias entre los imperialistas, estas colonias comienzan a acercarse a su imperio. El poder total de un imperio puede ser determinado por el poder del país imperialista más el porcentaje de poder de sus colonias, como sigue [5]:

$$
\text { power }_{n}=\operatorname{cost}\left(\text { imperialists }_{n}\right)+\varepsilon \operatorname{mean}\left\{\left(\operatorname{cost}\left(\operatorname{cost} \text { of empires }{ }_{n}\right)\right)\right\}
$$




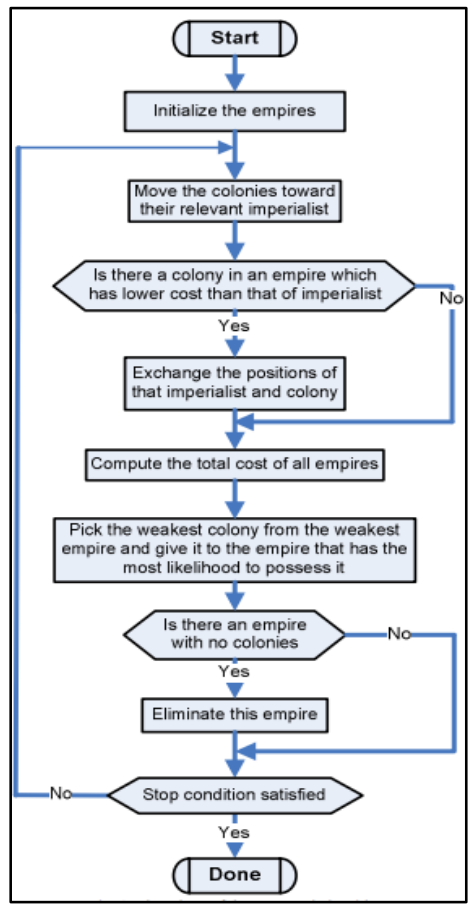

Fig. 1. Diagrama de flujo del ICA.

donde power $_{\mathrm{n}}$ es el poder total (costo) del n-ésimo imperio $\varepsilon$ es un número positivo menor que uno.

Los imperios más débiles colapsarán en la competencia imperialista y sus colonias se dividirán entre otros imperios. Después de un tiempo, todos los imperios, excepto el más poderoso, colapsarán y todas las colonias estarán bajo el control de este imperio [12], que es la respuesta del problema de optimización.

El desarrollo de un aparador comercial requiere, por un lado, del desarrollo conceptual y por otro, del desarrollo de diversas medidas de similitud que permitan establecer ubicaciones desde un concepto básico y en los datos. Pero es necesario priorizar el desarrollo conceptual y de las categorías del sistema del modelo visual, y paralelamente pensar en el modelo matemático.

Para este modelo, utilizamos la correlación para medir la similitud entre la ropa de una colección. Esta medida nos permite localizar cada prenda con respecto a otros y organizarla en grupos (diferentes temas en el aparador comercial). Un peso para cada atributo representa la importancia de un atributo. Para cada par de prenda se define la siguiente función de fitness:

$$
\sum_{\mathrm{i}}^{\mathrm{n}}=\operatorname{abs}\left[\operatorname{corr}\left(\mathrm{W}_{\mathrm{i}} \mathrm{Attribute}_{\mathrm{i}}, \mathrm{W}_{\mathrm{i}} \mathrm{Attribute}_{\mathrm{i}},\right)\right],
$$

donde: 


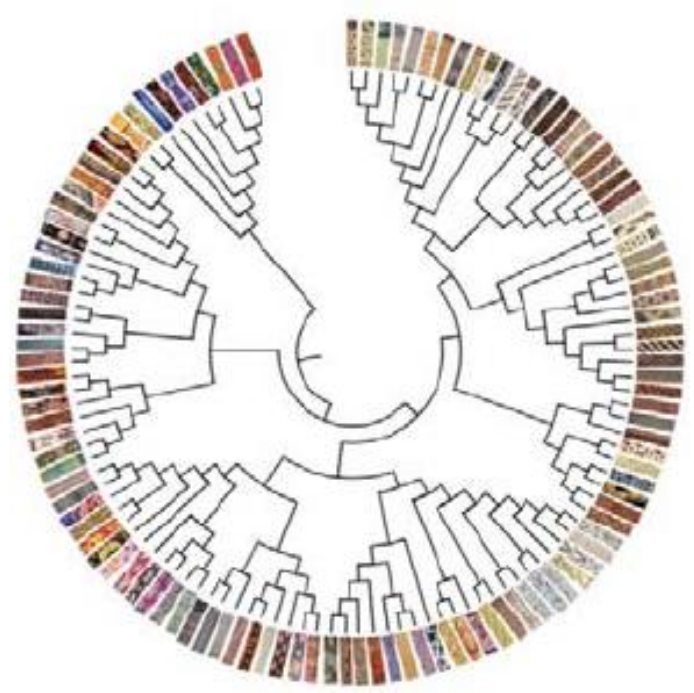

Fig. 2. Representación de colores a la ropa.

$\mathrm{W}_{\mathrm{i}}=$ Vector de pesos que representan la importancia del atributo $\mathrm{i}$,

$\mathrm{A}, \mathrm{B}=$ Representan las prendas (hombre-mujer) que se desea medir la similitud y el Attribute $_{\mathrm{i}}=$ Vector de los valores de los atributos de cada prenda.

Mientras que el valor de correlación disminuye, la relación entre las prendas es más fuerte. Se establece un umbral de 0.5 , si el valor de correlación es menor que el umbral, asumimos que no hay relación entre las prendas.

\section{Distribución de elementos basados en una adecuada representación visual de colores}

Desde el punto de vista de un algoritmo bio-inspirado, este problema de optimización combinatoria es muy complejo, debido a que no se conoce la mejor ubicación de un individuo representativo de una prenda en específico con respecto a los otros individuos [7]. En el algoritmo propuesto, los individuos en el beliefscape (beliefscape) a través de su mejor paradigma (BestParadigm) se ponen a cero para representar el hecho de que la cultura aumenta la expectativa asociada con la ubicación de una prenda con respecto a las demás y usar un modelo de representación social, incentivando el comportamiento asociado con el mejor paradigma (BestParadigm). En la figura 2 se muestra la representación de colores de las prendas con la selección más adecuada de temas y representación visual para distribuir elementos en un aparador comercial.

Para ello se seleccionó un almacén de prendas en existencia y se caracterizó su capital simbólico con base en ocho atributos que tiene una representación visual: control emocional, habilidad de lucha, inteligencia, agilidad, fuerza, resistencia, liderazgo social y velocidad, estas características permiten describir tanto a una 


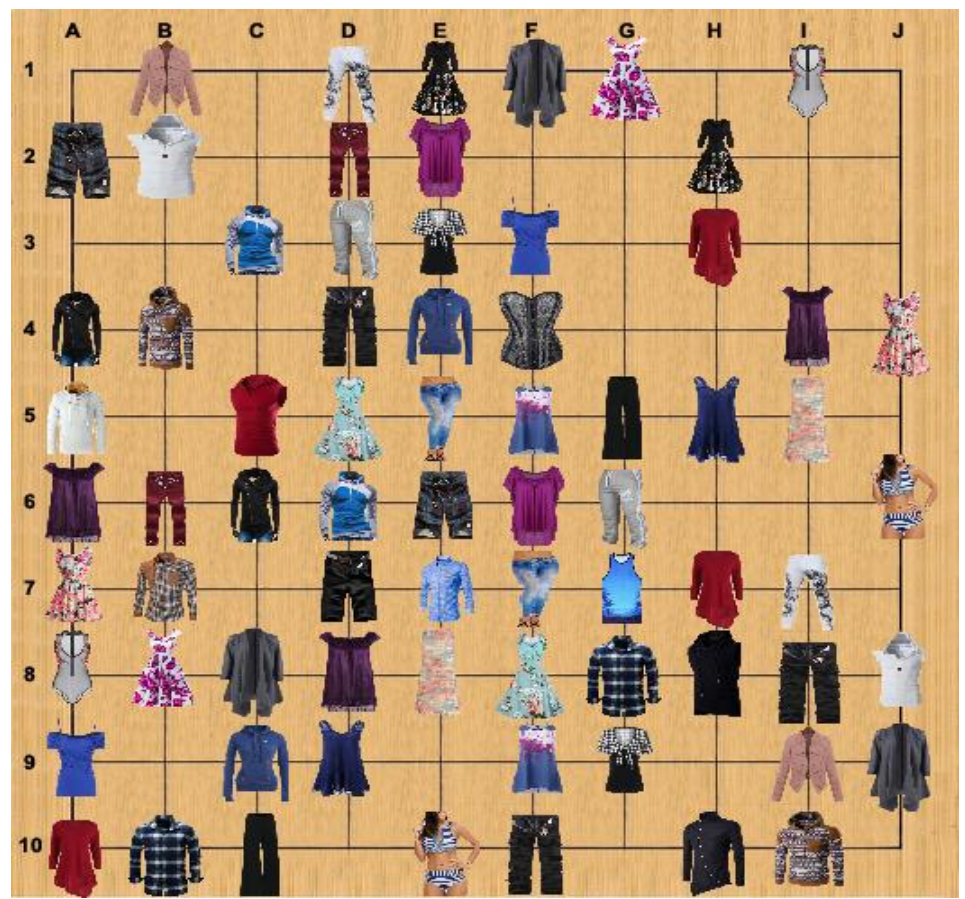

Fig. 3. Modelo comparativo para asignar ubicaciones en un tocador representado por prendas de mujer y de hombre.

sociedad como a la ropa asociada. La investigación está basada en el deseo de compartir la comprensión intuitiva sobre el tratamiento de una nueva clase de sistemas, individuos capaces de tener empatía, una característica reservada a las personas vivas. Desde la perspectiva de un modelo matemático, esto tiene varias restricciones relacionadas con el espacio, la combinación de colores y precio.

\section{Múltiples coincidencias}

Se realiza un rango de diecisiete evaluaciones según diferentes combinaciones de colores y vestuario de más de 50 ejecuciones en diferentes escenarios. El proceso de licitación para la selección final del armario para cada emisión comenzará cuando se analizan diferentes características. En aras del desarrollo proporcional, todas las prendas deben estar representadas, en la medida de lo posible y depender del interés generado en cada una. El momento de evaluar y contratar cada oferta de prendas será muy importante, especialmente al comienzo dela serie. Se dará preferencia a las cuestiones con más similitudes socioculturales.

Para competir se seleccionarán 87 temas. Cada número aceptado y se propondrá para participar en exactamente diecisiete de estas evaluaciones. Los temas evaluarán su preferencia de torneos, una vez que la lista final de múltiples coincidencias se evalúa y el algoritmo evalúa estos. El algoritmo se reserva el derecho de asignar prendas a 
evaluar de acuerdo a las necesidades de organización de su aparador comercial y las prendas para cada aparador comercial, así como asignar la lista de prendas antes de que comience el ciclo.

Cada evaluación tendrá hasta 88 prendas combinando sobre un programa de diecisiete evaluaciones con sus respectivas ejecuciones. Con el objetivo de armonización por parte del algoritmo, se programará para cumplir el programa de comparación de diferentes similitudes utilizando una ronda de análisis de concordancia múltiple y con base en el género asignado a un tema, como en la figura 3 .

Las prendas que califiquen para la selección en un aparador comercial serán elegidas teniendo en cuenta la siguiente prioridad:

\subsection{Coincidencias}

Para el primer ciclo de similitud, toda la ropa en el almacén y coincidencias de similitud se invitará a participar en diferentes comparaciones, tratar de determinar las dos prendas más similares a una persona. Dada la organización de cada una de las prendas que coincidan en cada ronda en el algoritmo, todas estas prendas se les pedirá que comprometan su participación en la evaluación de cada serie. En caso de que alguna de estas prendas deje de participar en la serie, el algoritmo puede designar una prenda para reemplazar y esta prenda debe ser clasificada en parte superior de la reposición del almacén, teniendo en cuenta esta situación como relevante, principalmente cuando el almacén es limitado.

\subsection{Clasificación}

Basándose en un cálculo promedio a dos decimales, la lista de calificación en la serie de comparaciones antes del inicio del ciclo, se seleccionarán veinte calificadores (excluyendo las siete prendas que se van a comparar las coincidencias). Si la prenda tiene la misma calificación promedio, se usará el número de similitudes evaluadas (período de calificación) para determinar la clasificación.

Para asegurar una participación en el futuro, se recomienda un mínimo de 25 combinaciones en las cuatro listas de calificación, incluyendo el valor de inicio de la lista de calificación. Dada la breve explicación de su evaluación y notificación en la primera edición de evaluaciones completas, se propone el uso de una guía narrativa para ello. Si alguna ropa no acepta jugar en la serie de múltiples coincidencias, entonces se adoptará el proceso de selección usando la calificación promedio más el número de juegos jugados en el período de calificación hasta que se complete el número de calificadores requeridos para la serie.

\section{Experimentación}

Para poder organizar el arreglo más eficiente de las prendas en un tocador, desarrollamos una atmósfera capaz de almacenar los datos de cada uno de los que representan estas prendas, esto con el fin de distribuir de una forma óptima a cada una 


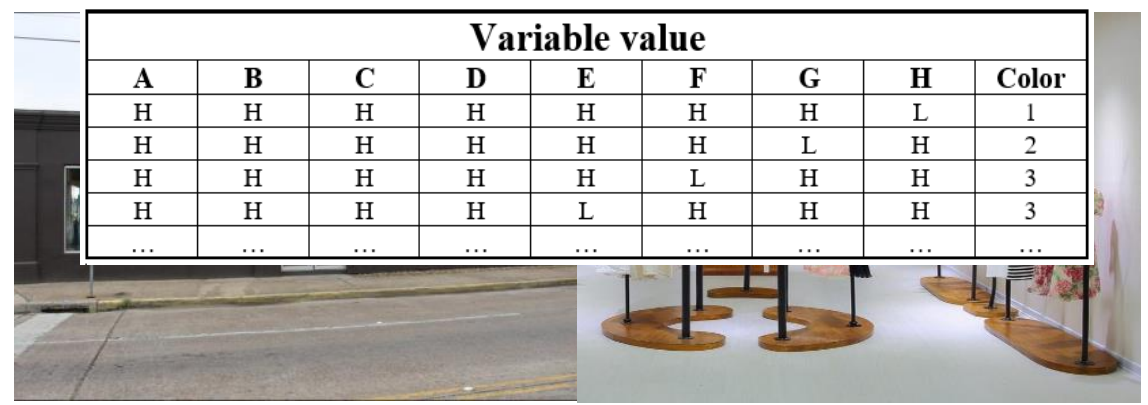

Fig. 4. Aparador final (interior-exterior) construido de acuerdo con hasta ocho atributos diversos basados en el Algoritmo Competitivo Imperialista (Sistema Multiagente).

de las prendas evaluadas. Una de las características más interesantes observadas en este experimento fue la diversidad de los patrones culturales establecidos por cada prenda con respecto a su capital simbólico.

Las escenas estructuradas asociadas con los agentes no se pueden reproducir en general, ya que sólo representan un poco de tiempo dados en el espacio y el tiempo de las diferentes prendas. Estos representan una forma única e innovadora de comportamiento adaptativo que resuelve un problema computacional que no intenta agrupar la prenda sólo con un factor asociado con su apariencia externa (atributos de cada atuendo), tratando de resolver un problema computacional que implica un cambio complejo entre las representaciones existentes.

Las configuraciones generadas pueden estar relacionadas con el conocimiento del comportamiento de un cliente potencial con respecto a un problema de optimización (para seleccionar culturalmente ropa similar, sin ser del mismo tipo [8]). El experimento principal consistió en detallar cada una de las prendas de una colección, con 500 agentes y una condición de 50 épocas, lo que nos permitió generar la mejor selección de cada tipo de prenda y su posible ubicación en un aparador comercial. Se obtuvo después de comparar las diferentes similitudes culturales y sociales de cada vestimenta, y evaluar con el modelo de múltiples coincidencias cada uno de ellos [9].

La investigación permitió clasificar cada una de las prendas pertenecientes a cada tipo, con diferente vestuario para prendas sólo con identidad cultural, lo que permite identificar cambios en el tiempo respecto a otras prendas. En la figura 4 se representa el aparador final (exterior-interior) construido según hasta ocho atributos diferentes, respectivamente (ver Figura 4).

El diseño del experimento consiste en una prueba de matriz ortogonal, con las interacciones entre las variables: control emocional, capacidad de lucha, inteligencia, agilidad, fuerza, resistencia, liderazgo social y velocidad. Estas variables se estudian en un rango de color (1 a 64). El arreglo ortogonal es $\mathrm{L}_{\mathrm{N}}(2 * * 8)$, en otras palabras, 8 factores en $\mathrm{N}$ ejecuciones, $\mathrm{N}$ se define por la combinación de los valores posibles de las 8 variables y el posible rango de color (Ver Figura 5). De acuerdo con los resultados obtenidos para el tiempo de alojamiento en el transporte debe realizarse para acomodar el tiempo de descarga en el siguiente letrero.

Sobre la base de los resultados obtenidos en el experimento, el promedio de similitud entre las prendas se encontró que el promedio es 4,0625; esto significa que debe tener 
que mejorar en la gestión del uso de la combinación de colores y estándar según el clima. Otros factores que afectan serían el uso de accesorios tales como: pulseras, collares, alfileres y encantos, una implementación para resolver este problema es el uso de la visualización de la moda. Como se puede ver para mantener este tipo de materiales es necesario utilizar la mercancía directa e indirecta. La mercancía indirecta asegura el suelo, da resistencia al movimiento lateral y longitudinal de la ropa. Mientras que las corbatas directas ancladas a los maniquíes en la cómoda. Para realizar este proceso, es necesario contar con las dimensiones correctas y la distancia de la calle y la altura de estos, cables o cadenas, teniendo en cuenta el material de estos maniquíes.

\section{Conclusiones}

Mediante la utilización del ICA se mejora el entendimiento para obtener el cambio del "mejor paradigma", porque se clasifica apropiadamente las comunidades de agentes al basarse en un acercamiento a la relación que mantienen sus atributos, esto permitió entender que el concepto de "moda basada en el capital simbólico" existe con base en la determinación de la función de aceptación por parte del resto de la ropa al lugar propuesto para el resto de los mismos. ICA ofrece una poderosa alternativa a los problemas de optimización y redistribución de la técnica de clustering. Por esta razón, esta técnica ofrece un panorama bastante comprensible del fenómeno cultural representado [6]. Esta técnica permite incluir la posibilidad de generar conocimiento experimental creado por la comunidad de agentes para un nuevo dominio de aplicación.

El análisis del nivel y grado de conocimiento cognitivo de cada comunidad es un aspecto que se desea evaluar para el trabajo futuro. La respuesta puede residir entre la similitud que existe en la comunicación entre dos culturas diferentes y como estas son percibidas [10].

Por otro lado, comprender las verdaderas similitudes que tienen diferentes sociedades con base en las características que las hacen contribuyentes de cluster y le permite también mantener su propia identidad, demuestra que las pequeñas variaciones van más allá de las características fenotípicas y son principalmente asociadas a gustos y características similares desarrolladas a través del tiempo [11]. Una nueva Inteligencia Artificial puede tener cuidado de analizar al por menor estas complejidades que cada sociedad mantiene, sin olvidar que aún nos necesitan métodos para entender las características originales y particulares de cada sociedad.

Agradecimientos A la comunidad de Sociología Computacional que durante una década ha vuelto a buscar formas novedosas de mostrar representación visual y desarrollado diferentes Ateliers of Artificial Societies.

\section{Referencias}

1. Das, T. K., Venayagamoorthy, G. K., Aliyu. U. O.: Bio-inspired algorithms for the design of multiple optimal power system stabilizers: SPPSO and BFA. IEEE Trans. Ind. Appl., pp. 1445-1457 (2008) 
2. Back, T.: Evolutionary Algorithms in Theory and Practice: Evolution Strategies, Evolutionary Programming, Genetic Algorithms. (1996)

3. Colorni, A., Dorigo, M., Maniezzo, V.: Distributed Optimization by Ant Colonies. In: Actes de la première conférence européenne sur la vie artificielle, pp. 134-142 (1991)

4. Kennedy, J., Eberhart, R.: Particle Swarm Optimization. In: Proceedings of IEEE International Conference on Neural Networks, Washington (1995)

5. Atashpaz-Gargari, E., Lucas, C.: Imperialist competitive algorithm: an algorithm for optimization inspired by imperialistic competition. In: IEEE Congress on evolutionary computation, Singapore (2007)

6. Zuckermann, D.: Culture and Organizations.

7. Ochoa, A. et al.: Baharastar - Simulador de Algoritmos Culturales para la Minería de Datos Social. In: Proceedings of COMCEV'2007 (2007)

8. Memory Alpha: Memory-alpha.org (Star Trek World) (2009)

9. Vukčević, I., Ochoa, A.: Similar cultural relationships in Montenegro. In: JASSS'2005 (2005)

10. Ponce, J. et al.: Data Mining and Knowledge Discovery in Real Life Applications. In: Book edited by: Julio Ponce and Adem Karahoca (2009)

11. Ustaoglu, Y.: Simulating the behavior of a minority in Turkish Society. In: ASNA'2009 (2009)

12. Bernal, E., Castillo, O., Soria, J., Valdez, F.: Imperialist Competitive Algorithm with Dynamic Parameter Adaptation Using Fuzzy Logic Applied to the Optimization of Mathematical Functions. pp. 1-19 (2017) 